\title{
Topical nepafenac in the treatment of diabetic macular edema
}

\author{
David Callanan \\ Patrick Williams \\ Texas Retina Associates, \\ Arlington, TX, USA
}

Correspondence: David Callanan Texas Retina Associates, I00I N Waldrop Dr Suite 512 , Arlington, TX 76012, USA $\mathrm{Tel}+18172619625$

Fax + 8172619586

Email dcallanan@texasretina.com
Purpose: To determine the safety and efficacy of topical nepafenac $0.1 \%$ in the treatment of diabetic macular edema.

Methods: A consecutive case series was performed of patients treated with nepafenac monotherapy twice daily for diabetic macular edema. Visual acuities (VA) at baseline and final visit were recorded. Foveal thickness, based on optical coherence tomography, was also recorded.

Results: Six eyes of 5 patients were included in this study. Median initial Snellen vision was 20/100 (range, 20/40-20/400). After a mean of 210 days (range, 182-259), median final VA was 20/75 (range, 20/40-20/400). Four eyes gained vision and two eyes maintained vision. Mean pre-treatment vision was $0.78 \log$ MAR and final VA was $0.67 \log \mathrm{MAR}$, for a statistically significant improvement ( $\mathrm{p}<0.05$ ). Mean initial foveal thickness was 417 microns (range, 286-599). After a mean of 178 days (range, 91-259), mean foveal thickness was 267 microns (range, 158-423), showing a statistically significant improvement $(\mathrm{p}<0.05)$. Each eye had an improvement in foveal thickness.

Conclusion: The results from these 6 eyes suggest that nepafenac $0.1 \%$ may have activity against diabetic macular edema and warrants further investigation.

Keywords: diabetes, diabetic macular edema, inflammation, macular edema, nepafenac, NSAIDs

\section{Introduction}

Multiple treatment options exist for diabetic macular edema. The safety and efficacy of focal laser treatment for clinically significant macular edema was established by the Early Treatment for Diabetic Retinopathy Study (ETDRS 1985). More recently, intravitreal injections have become increasingly popular among clinicians. Based on published reports, both triamcinolone and bevacizumab have had success in reducing edema and improving vision (Gillies et al 2006; Haritoglou et al 2006).

Each treatment available has some drawbacks. While focal laser treatment can reduce the odds of vision loss and improve the odds of vision gain (ETDRS 1985), the actual reduction in edema and improvement in vision can sometimes be less than desired. Also, microaneurysms at the edge of the foveal avascular zone can be difficult to treat due to the fear of permanent scotoma. In contrast, intravitreal injections tend to generate a rapid reduction in edema (Gillies et al 2006; Haritoglou et al 2006). However, each intravitreal injection carries a risk of endophthalmitis (Gillies et al 2006; Haritoglou et al 2006). In addition, intravitreal steroids carry the additional risks of glaucoma and cataract formation (Gillies et al 2006).

For cases of mild macular edema, the risks of established treatments may outweigh the benefits. Thus, alternative treatments with better safety profiles are desirable. Because elevated inflammatory markers have been found in patients with 
diabetic retinopathy, it is likely that inflammation aids in the progression of vascular disease in these patients ( $\mathrm{Ke}$ et al 2000; Meleth et al 2005). For this reason, nonsteroidal antiinflammatory drugs (NSAIDs) may be effective against diabetic macular edema. Topical NSAIDs are attractive because they have few documented risks. In addition, the newer topical NSAIDs have a theoretical potential to reduce vascular permeability in patients with diabetic macular edema. Nepafenac is a prodrug that is hydrolyzed into amfenac by uveal tissue and retina (Ke et al 2000). Based on animal models, nepafenac has the capability to penetrate into the posterior segment (Kern et al 2007; Kapin et al 2003). If an NSAID can penetrate to the retina, it could possibly reduce vascular permeability by inhibiting the inflammatory cascade. Based on this hypothesis, patients with diabetic macular edema were started on topical nepafenac $0.1 \%$ to attempt to reduce the edema and improve vision. This study is a review of these patients with diabetic macular edema treated with nepafenac (Nevanac; Alcon Labs, Fort Worth, TX, USA).

\section{Methods}

A consecutive case series of all patients using nepafenac monotherapy for diabetic macular edema was performed. Seven eyes of six patients were evaluated for inclusion. One eye was excluded due to corneal transplantation for unrelated Fuch's dystrophy. Inclusion criteria included evidence of diabetic retinopathy (microaneurysms, intraretinal hemorrhages, hard exudates, cotton wool spots), macular edema demonstrated by optical coherence tomography (OCT), use of topical nepafenac, and at least six months follow-up. Any patient with an intravitreal injection or focal laser treatment within the previous six months was excluded. No patient had cataract surgery within five years of the study period. Any patient with vision loss not solely attributed to diabetic retinopathy was excluded from analysis. Demographic data as well as previous treatments were logged. Visual acuities at baseline and at the final visit were recorded. Foveal thickness, based on OCT, was also recorded at baseline and final visit. A one-tailed paired t-test was used to compare pre- and post-treatment foveal thickness and pre-and post-treatment visual acuity. All patients were maintained on twice a day nepafenac throughout the duration of the study.

\section{Results}

Charts of seven eyes of six patients who were consecutively started on nepafenac were evaluated for inclusion. One eye was excluded upon the initial chart review due to corneal transplantation for unrelated Fuch's dystrophy during the study period. Six eyes of five patients were included for visual and OCT analysis. Patients 1,3 , and 4 were phakic and patients 2 and 5 underwent cataract surgery five years prior to the study period.

The median initial Snellen vision was 20/100 (range, 20/40-20/400). After a mean of 210 days (range, 182-259), the median final visual acuity was 20/75 (range, 20/40-20/400). Four eyes gained vision, and two eyes maintained vision. No eye had a decrease in visual acuity. Visual acuities were converted to $\log$ MAR equivalent for statistical analysis. The mean pre-treatment vision was $0.78 \log$ MAR and the final visual acuity was $0.67 \log$ MAR. The average improvement was statistically significant based on a one-tailed paired t-test $(\mathrm{p}<0.05)$.

The mean initial foveal thickness was 417 microns (range, 286-599). After a mean of 178 days (range, 91-259), the foveal thickness was 267 microns (range, 158-423). The mean duration of treatment for OCT analysis was less than that of the visual analysis because OCT was not performed at every visit. Each eye had an improvement in foveal thickness; Figure 1 shows the pre- and post-treatment OCT data for patient \#3. The average improvement was statistically significant based on a one-tailed paired t-test $(\mathrm{p}<0.05)$. The differences in vision and OCT are demonstrated in Table 1.

Table 1 also shows the hemoglobin A1c values for the patients at the beginning of treatment with nepafenac and at the time of the final OCT readings. Patient 5 did not have regular $\mathrm{HbA} 1 \mathrm{c}$ values available. None of the other patients had evidence of improved glycemic control during the study period as measured by $\mathrm{HbA} 1 \mathrm{c}$ and patients 3 and 4 actually had a deterioration in their glycemic control over the study period. One patient developed a vitreous hemorrhage seven months after the study period that was not considered related to nepafenac use. No complications such as keratitis that can be caused by topical nonsteroidal drops were noted.

\section{Discussion}

The results of this pilot study suggest a benefit of topical nepafenac in the treatment of diabetic macular edema. One limitation of the study is the absence of matched controls. There did not, however, appear to be a significant change in the systemic diabetes status of the patients during the study period. All of the patients had a reduction in foveal thickness in the treated eye on OCT. This is consistent with the results of nepafenac treatment in a rabbit model of macular edema 


\section{A}

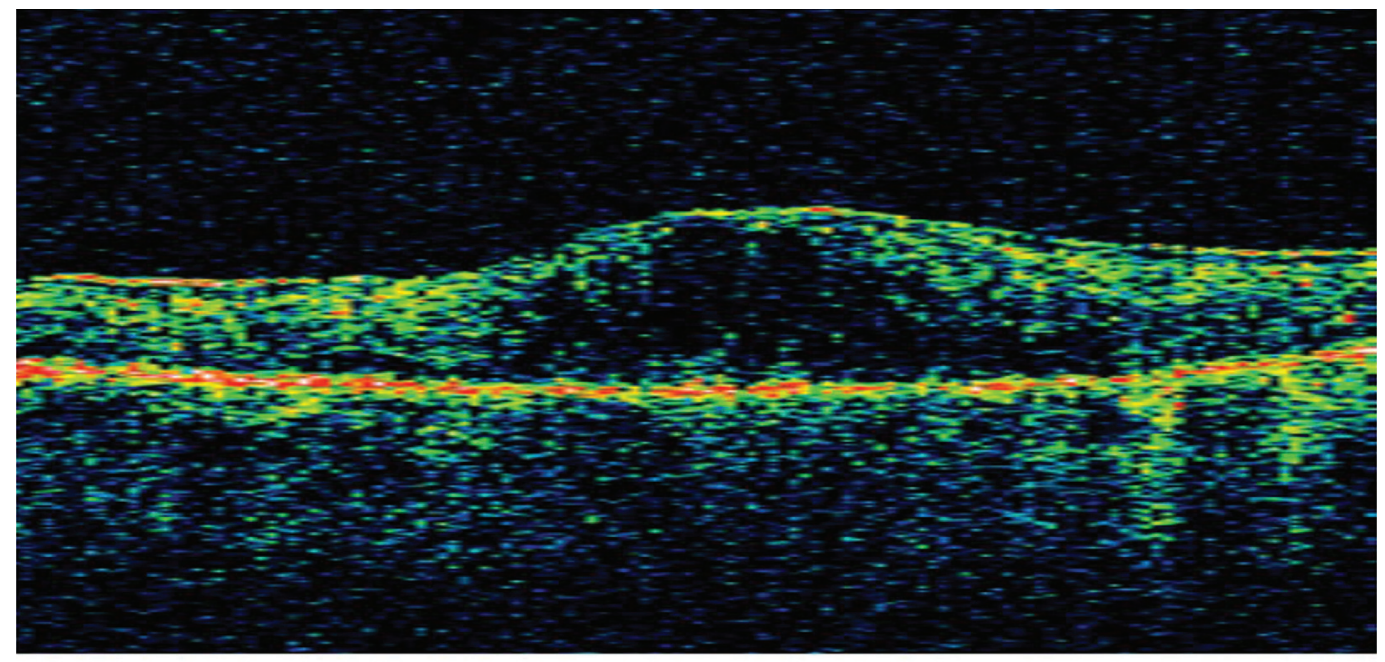

\section{B}

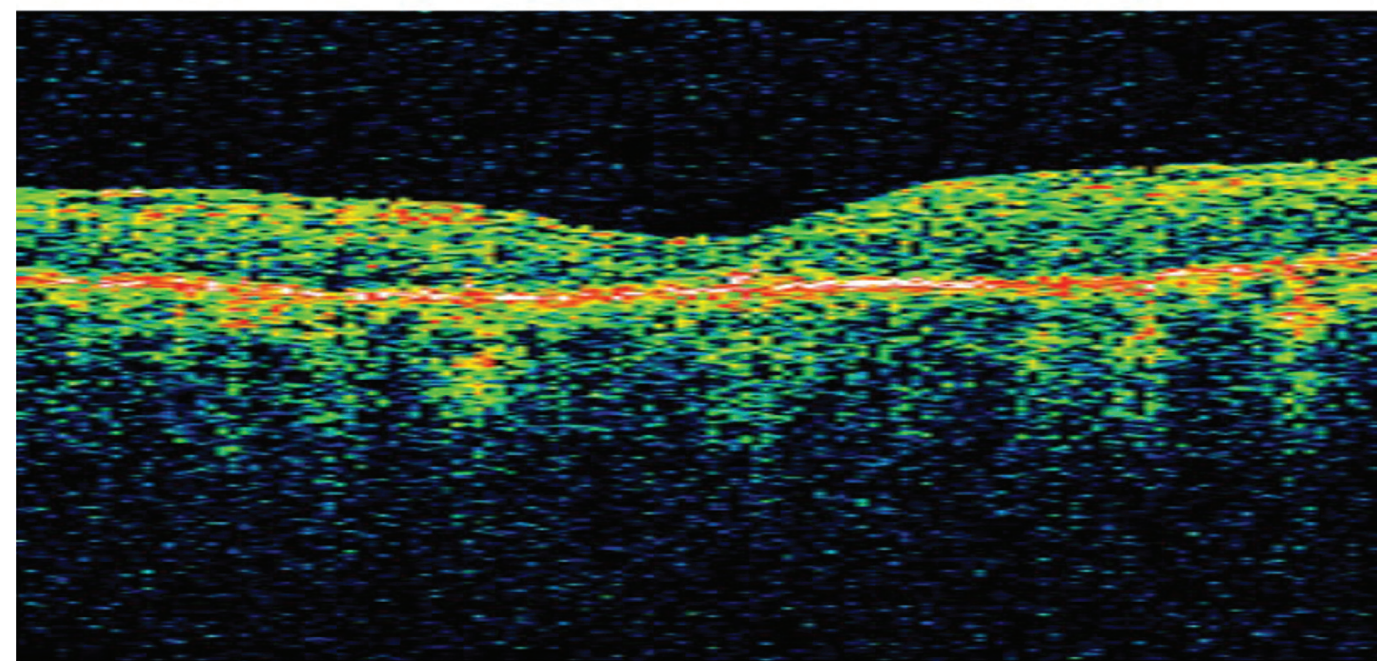

Figure I A OCT image of left eye in Patient \#3 at baseline. Central subfield thickness was $517 \mu \mathrm{m}$ and total volume was $9.02 \mathrm{~mm}^{3}$. B OCT image of left eye in Patient \#3 after 259 days of nepafenac treatment. Central subfield thickness was I $58 \mu \mathrm{m}$ and total volume was $7.35 \mathrm{~mm}^{3}$.

(Kapin et al 2003). Antiinflammatory treatment has been proposed in the past as a mechanism for slowing diabetic changes in the retina. Sustained delivery of steroids in human diabetic eyes using a fluocinolone implant has been shown to improve diabetic retinopathy scores (Pearson and Levy 2005).
An initial trial of oral celecoxib in humans as adjunctive treatment for diabetic macular edema showed reduced retinal leakage as measured by fluorescein angiography (E Chew pers comm). Animal models of diabetic macular edema have also shown improvement using NSAIDs. A study using diabetic

Table I Demographics, visual acuity, foveal thickness, and hemoglobin Alc (HbAlc) results before and after nepafenac treatment

\begin{tabular}{lllllllllll}
\hline Patient & $\begin{array}{l}\text { Age } \\
\text { sex }\end{array}$ & Eye & $\begin{array}{l}\text { Baseline } \\
\text { vision }\end{array}$ & $\begin{array}{l}\text { Final } \\
\text { vision }\end{array}$ & $\begin{array}{l}\text { Days to final } \\
\text { vision }\end{array}$ & $\begin{array}{l}\text { Baseline foveal } \\
\text { thickness }(\mu \mathrm{m})\end{array}$ & $\begin{array}{l}\text { Final foveal } \\
\text { thickness }(\mu \mathrm{m})\end{array}$ & $\begin{array}{l}\text { Days to final } \\
\text { foveal thickness }\end{array}$ & $\begin{array}{l}\text { Initial } \\
\text { Hbalc\% }\end{array}$ & $\begin{array}{l}\text { Final } \\
\text { HbAlc\% }\end{array}$ \\
\hline I & $65 \mathrm{M}$ & OS & $20 / 80$ & $20 / 50$ & 193 & 378 & 215 & 193 & 7.0 & 6.8 \\
2 & $68 \mathrm{M}$ & OS & $20 / 40$ & $20 / 40$ & 209 & 286 & 226 & 209 & 6.5 & 6.8 \\
3 & $66 \mathrm{~F}$ & OS & $20 / 240$ & $20 / 140$ & 259 & 517 & 158 & 259 & 7.3 & 9.4 \\
4 & $68 \mathrm{~F}$ & OS & $20 / 120$ & $20 / 80$ & 227 & 599 & 423 & 227 & 4.9 & 6.0 \\
5 & $76 \mathrm{M}$ & OD & $20 / 80$ & $20 / 70$ & 182 & 342 & 267 & 91 & \\
5 & & OS & $20 / 400$ & $20 / 400$ & 182 & 380 & 310 & 91 & & \\
\hline
\end{tabular}


rats provides some insight into the potential mechanism of action of nepafenac. Kern and colleagues discovered that daily topical treatment with nepafenac produced significant declines in diabetes-induced biochemical alterations, including retinal prostaglandin $\mathrm{E}_{2}\left(\mathrm{PGE}_{2}\right)$, cyclooxygenase-2 (COX2), and superoxide production (Kern et al 2007). In addition, cellular and morphologic changes such as leukostasis, retinal capillary degeneration, and endothelial cell apoptosis were inhibited by nepafenac. Thus, it appears that nepafenac has a positive effect on diabetes-induced ocular pathology.

While a majority of patients in the current study had some improvement in vision, two patients had an anatomic improvement without a gain in visual acuity. This may be due to retinal ischemia causing cellular damage unresponsive to edema resolution. As OCT becomes more commonplace in judging success of treatment for retinal edema, it will be important to predict those with the potential for visual improvement. A previous study described the importance of OCT reflectivity of the inner retinal layers in predicting visual improvement along with anatomic improvement (Gibran et al 2007). More studies are required to determine those patients more likely to gain vision with resolution of macular edema. A subset of diabetics has relatively good vision and mild cystic changes on OCT. These patients may be more likely to maintain their vision with correction of this mild cystic edema. Although corneal morbidities such as keratitis are possible with long term use of topical nonsteroidals, no patient in this small series had a complication attributed to nepafenac treatment.

This small case series suggests a benefit from topical nepafenac. Considering the safety profile of topical nepafenac, a study is warranted to examine the role of nepafenac in preventing vision loss due to diabetic macular edema in these patients. A larger, controlled, and randomized trial is needed and is being planned.

\section{Disclosure}

This research was previously presented at the 2007 Annual Meeting of the Association for Research and Vision in Ophthalmology, May 6-10, 2007, abstract \#5001. Neither author has a propriety interest in the outcome of this study. Dr Callanan is a member of an advisory board for Alcon Laboratories, Inc.

\section{References}

[EDTRS] Early Treatment Diabetic Retinopathy Study research group. 1985. Photocoagulation for diabetic macular edema. Early Treatment Diabetic Retinopathy Study report number 1. Arch Ophthalmol, 103:1796-806.

Gibran SK, Khan K, Jungkim S, et al. 2007. Optical coherence tomographic pattern may predict visual outcome after intravitreal triamcinolone for diabetic macular edema. Ophthalmology, 114:890-4.

Gillies MC, Sutter FK, Simpson JM, et al. 2006. Intravitreal triamcinolone for refractory diabetic macular edema: two-year results of a doublemasked, placebo-controlled, randomized clinical trial. Ophthalmology, 113:1533-8.

Haritoglou C, Kook D, Neubauer A, et al. 2006. Intravitreal bevacizumab (Avastin) therapy for persistent diffuse diabetic macular edema. Retina, 26:999-1005.

Kapin MA, Yanni JM, Brady MT, et al. 2003. Inflammation-mediated retinal edema in the rabbit is inhibited by topical nepafenac. Inflammation, 27:281-91.

Ke TL, Graff G, Spellman JM, et al. 2000. Nepafenac, a unique nonsteroidal prodrug with potential utility in the treatment of trauma-induced ocular inflammation: II. In vitro bioactivation and permeation of external ocular barriers. Inflammation, 24:371-84.

Kern TS, Miller CM, Du Y, et al. 2007. Topical administration of nepafenac inhibits diabetes induced retinal microvascular disease and underlying abnormalities of retinal metabolism and physiology. Diabetes, 56:373-9.

Meleth AD, Agron E, Chan CC, et al. 2005. Serum inflammatory markers in diabetic retinopathy. Invest Ophthalmol Vis Sci, 46:4295-301.

Pearson PA and Levy B. 2005. Intravitreal fluocinolone implant for diabetic macular edema: Two year results. ARVO Annual Meeting, Ft. Lauderdale, FL, May 1-5. 